\title{
Spatial multistability induced by cross interactions of confined polariton modes
}

\author{
C. Ouellet-Plamondon, , G. Sallen, F. Morier-Genoud, D. Y. Oberli, M. T. Portella-Oberli, and B. Deveaud \\ Institute of Physics, École Polytechnique Fédérale de Lausanne (EPFL), CH-1015 Lausanne, Switzerland \\ (Received 16 December 2015; revised manuscript received 10 February 2016; published 29 February 2016)
}

\begin{abstract}
We demonstrate the occurrence of spatial multistability using laterally confined microcavity exciton-polaritons. By coherently exciting with a blue detuned laser a series of confined polariton modes, we investigate the effects of multistability on the transmitted laser beam as a function of the excitation power. At each threshold of the hysteresis loop, a switching of the mode profile of the laser beam is associated with a significant energy jump of each of the confined polariton modes in the mesa. A simulation of this behavior is achieved with a multimode generalization of the Gross-Pitaevskii equations in the exciton photon basis. The mechanism behind the spatial multistability is identified as a repulsive cross interaction between polaritons in different modes.
\end{abstract}

DOI: 10.1103/PhysRevB.93.085313

\section{INTRODUCTION}

An optically driven system is said to be bistable if there exist two possible output intensities over a range of input powers [1]. When more than two output intensities are allowed either when cycling the input power or for a given input value, the result is an optical multistability, as was observed for example for atomic systems in an optical resonator [2-5]. There exists a different kind of optical multistability called spatial multistability which consists of having specific transverse spatial profiles for each of the stable states of the optical multistability. This effect was first demonstrated using a $\mathrm{HeNe}$ laser cavity [6], as well as other gas lasers [7,8], and was theoretically discussed in Refs. [9,10]. It relies on being able to select and switch between laser cavity modes out of a superposition of modes either by injecting the right phase pattern or by displacing a saturable absorber inside the cavity.

In this work we present the demonstration of spatial multistability based on confined microcavity exciton-polaritons. By laterally confining light either by engineering the microcavity [11-14], or by the use of oval defects [15], it is possible to create a series of spatially distinct, confined polariton modes when these new cavity modes are strongly coupled to an exciton in a quantum well (QW). Therefore, taking advantage of the nonlinearity in the optical response of the polariton modes resulting from the exciton-exciton interactions, we can coherently excite this system to obtain the spatial multistability simply by changing the excitation power. In contrast with the mode competition in laser cavity where mode switching occurs without a change of the output power, the spatial mode switching of the polariton spatial multistability is associated with an intensity threshold, as a consequence of polaritonpolariton interactions.

The bistability and spinor multistability of a microcavity polariton have been extensively studied both theoretically [16-22] and experimentally [23-32]. It relies on exciting the microcavity using a laser blue detuned with respect to the lower polariton branch (LP) and to measure the transmitted laser intensity. When cycling the excitation power, an hysteresis curve is observed that is a consequence of the Kerr-like nonlinearity induced by exciton-exciton interactions [23].

\footnotetext{
*clauderic.ouellet-plamondon@epfl.ch
}

Specifically, the spinor multistability is achieved by rotating the polarization of the input laser on the Poincaré sphere to trigger the polariton spinor interactions in order to obtain a multistable pseudospin system [24]. This effect is caused by the population of one polariton spin state that can induce a blue shift in the orthogonally polarized polariton branch [33]. Then, the overall competition between the excitation laser polarization, spinor interactions, and cavity anisotropy dictates the possible outcome of the multistability [28,31,33].

In our study we demonstrate that polariton-polariton interactions occur between confined polariton modes. We observe that the population in one mode induces a blue shift on the other modes when increasing the resonant excitation power by measuring weak photoluminescence (PL) signals below and above the excitation laser energy. The mechanism involved in the observed spatial multistability can be seen as a generalization of the spinor multistability, that is, a cross interaction of more than two independent polariton modes. At low density, the polarized polariton states are orthogonal as a consequence of in-plane momentum conservation of the exciton-photon interaction [34,35]. At high enough density, these polaritons start to interact due to the spin dependence of the exciton-exciton interactions. For our circular traps, the confined polariton modes are initially orthogonal due to the orthogonality of the photon modes [35,36]; however, they evolve with increasing density since they originate from the same exciton state. We confirm this theoretically by extending the usual formalism of spinor Gross-Pitaevskii equations, in order to describe our multimode interacting system. We obtain a set of coupled differential equations in the exciton-photon basis that adequately simulate the strong coupling of excitons and confined photons inside the mesa and accounts for the existence of cross interactions.

\section{SAMPLE AND EXPERIMENTAL METHOD}

The sample studied was grown by molecular beam epitaxy (MBE) and consists of a 21 (22) top (bottom) GaAs/AlAs distributed Bragg reflector (DBR) $\lambda$ microcavity with a single $\mathrm{In}_{0.04} \mathrm{Ga}_{0.96} \mathrm{As}$ QW placed at the antinode of the electromagnetic field inside the cavity spacer with a vacuum Rabi splitting of $\Omega_{0}=3.5 \mathrm{meV}$ [11]. The spacer has been chemically etched before the overgrowth of the top DBR to create circular mesas of $6 \mathrm{~nm}$ in height and 3 to $20 \mu \mathrm{m}$ 
TABLE I. Energy, cavity detuning $\delta$, and excitonic fraction $|X|^{2}$ of the first five polariton states of the studied $9 \mu \mathrm{m}$ mesa. $\Delta E_{\mathrm{LP}}=$ $E_{i}-E_{1}$ is the polariton energy relative to the energy $E_{1}$ of the ground state. The cavity detuning for the $i$ th polariton state is defined as $\delta=E_{c i}-E_{x}$, where $E_{c i}$ is the energy of the $i$ th cavity mode and $E_{x}$ is the exciton energy.

\begin{tabular}{lcccc}
\hline \hline$E_{i}$ & Energy (eV) & $\Delta E_{\mathrm{LP}}(\mathrm{meV})$ & $\delta(\mathrm{meV})$ & $|X|^{2}$ \\
\hline 1 & 1.48235 & 0.00 & -0.73 & 0.40 \\
2 & 1.48248 & 0.13 & -0.50 & 0.43 \\
3 & 1.48260 & 0.25 & -0.29 & 0.46 \\
4 & 1.48284 & 0.50 & 0.18 & 0.53 \\
5 & 1.48297 & 0.60 & 0.47 & 0.57 \\
\hline \hline
\end{tabular}

in diameter that act as a confinement potential $(9 \mathrm{meV})$ for light [37]. The resulting number of cavity modes for a specific mesa depends on its diameter and defines the number of confined polariton modes (one lower and one upper branch for each cavity mode, see Sec. IV). In the present study we use a mesa of $9 \mu \mathrm{m}$ in diameter where the fundamental cavity mode has a detuning of $\delta=-0.73 \mathrm{meV}$ with respect to the exciton state $(1.4845 \mathrm{eV})$ and a corresponding polariton ground state excitonic fraction of $|X|^{2}=0.40$. The measured linewidth of this mode is FWHM $=115 \mu \mathrm{eV}$ corresponding to a $Q$ factor of about 13000 . The energy, excitonic fraction, and respective cavity detuning for the excited polariton states are given in Table I. The spatial distribution of these confined states can be visualized by nonresonant PL either by energy resolving a slice of the spatial profile as shown in Fig. 1(a), or by performing a full tomography of the states, as explained below. With this procedure, we image the polariton ground
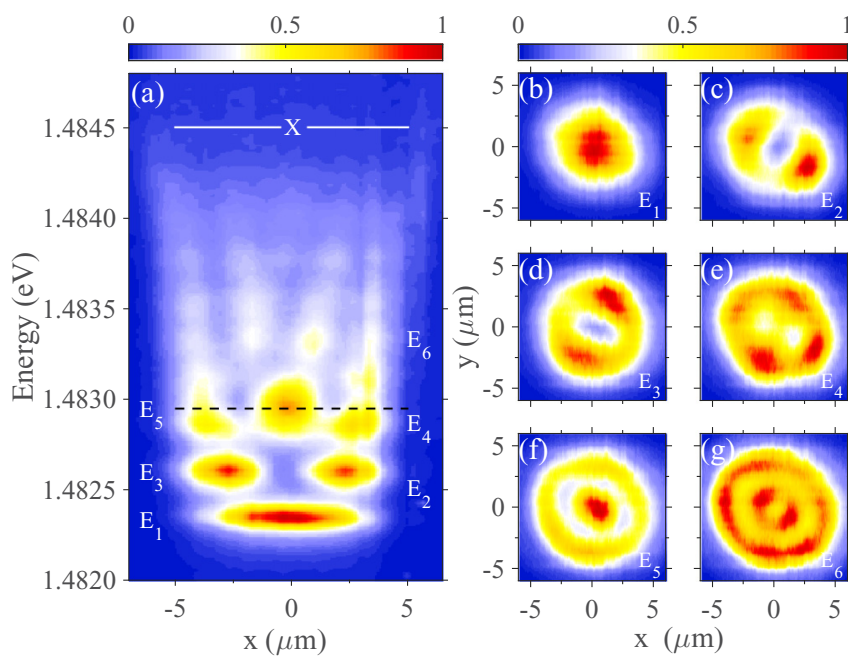

FIG. 1. (a) PL intensity as function of energy and position for a $9 \mu \mathrm{m}$ mesa under nonresonant excitation. The black dashed line indicates the energy position of the excitation laser for the multistability experiment and the $\mathrm{x}$ marked white line is the position of the bare exciton. (b)-(g) Spatial profile of the first six confined states starting from the ground state labeled $E_{1}$ to $E_{6}$. All intensities are normalized and shown in linear color scale. The first excited state $E_{2}$ in (c) is hidden in (a) due to the position of the slit cutting the spatial profile. state (labeled $E_{1}$ in Sec. IV) in Fig. 1(b) and the first five excited states in Figs. 1(c) -1 (g) (labeled $E_{2}$ to $E_{6}$ ). Since the trap is elliptical, the full circular symmetry is broken, which lifts the degeneracy of the states of angular quantum number $m$ for a given radial quantum number $n[35,38]$. Hence, the first two excited states $E_{2}$ and $E_{3}$ correspond to the $(n=1, m=1)$ doublet [Figs. 1(c) and 1(d)] and the excited state $E_{4}$ in Fig. 1(e) corresponds to a superposition of the even and odd solutions of the $(n=2, m=2)$ state, the energy splitting of which is less than our spectral resolution $(70 \mu \mathrm{eV})$ [38].

The experimental method allows the observation of the polariton bistability and can be summarized as follows [23,24]. First, the microcavity is driven by a laser blue detuned with respect to the LP mode. As the excitation power increases, the LP mode shifts upward in energy due to polariton-polariton repulsive interaction and a strong nonlinearity occurs once the shift compensates the laser detuning. Then, as the excitation power is decreasing, the LP remains locked to the laser down to a critical density where a lower threshold occurs. With the same reasoning, we can expect that, if the system is driven by a laser blue detuned with respect to $N$ polariton modes without cross interactions, then we should observe the superposition of $N$ independent bistable loops, with each polariton mode getting locked to the laser once their energy equals that of the laser. Since each polariton mode has a particular spatial profile, the spatial profile of the transmitted laser at each stable branch on the multistability will be the sum of the profiles of each of the states locked to the laser at that excitation power. However, if repulsive cross interaction exists between the confined polariton modes, then a polariton mode that blue shifts up to the laser energy should expel the former state locked to the laser. Having a single polariton mode locked to the laser on each stable branch of the multistability will result in the spatial multistability. As shown below, our experimental findings are well explained with this simple picture and, thus, it allows us to investigate cross level interactions.

In order to observe the spatial multistability, the energy spacing between the polariton modes should not be too large, otherwise polariton lasing might switch on when increasing the input power before the multistability occurs. This was observed in $3 \mu \mathrm{m}$ mesa [39] and is a consequence of efficient phonon scattering in the mesa [40] and the onset of bosonic stimulation when the occupation of the ground state reaches the critical density. For this reason the experiments are performed on a $9 \mu \mathrm{m}$ mesa instead of a $3 \mu \mathrm{m}$ one.

In our experiments, we excite resonantly with a single mode $\mathrm{cw}$ Ti:sapphire laser the fifth polariton state $\left[E_{5}\right.$ situated at around $1.48297 \mathrm{eV}$, see Fig. 1(a)], well below the fundamental cavity mode (situated at $1.48378 \mathrm{eV}$ ) to maintain the strong coupling regime at high density. This state is chosen because its large component at $k=0$ allows for an efficient pumping of the system and its detuning allows us to observe the nonlinearity of the polariton ground state before reaching our maximum excitation power. We detect the transmitted signal either with a power meter to integrate the full spatial profile or by focusing the beam on the slit of a spectrometer equipped with a CCD camera. With a proper choice of focal length, we create an image larger than the size of the entrance slit. Therefore, by translating the focusing lens in front of the spectrometer, we can perform an energy resolved tomography and recover the 
full spatial profile for a given energy as shown in Figs. 1(b)$1(\mathrm{~g})$. The excitation spot size of $\phi \approx 30 \mu \mathrm{m}$ creates a uniform excitation of the $9 \mu \mathrm{m}$ mesa and the sample is kept at $\sim 4 \mathrm{~K}$ in a helium flow cryostat. The excitation laser is circularly polarized, and the detection is unpolarized.

\section{SPATIAL MULTISTABILITY}

Figure 2 presents the multihysteresis cycle resulting from the excitation scheme explained above while detecting with the power meter (spatially integrating the signal). As the excitation power is increasing (following the red arrows), we measure three distinct upward jumps of the transmitted intensity, whereas four downward jumps are observed when the power is decreasing (blue arrows). A small nonlinearity is also visible on the way up at around $4 \mathrm{~mW}$. To highlight the spatial multistability, we present the spatial transverse profiles of the transmitted signal at the energy of the laser for selected power positions as indicated by the black arrows in Fig. 2. By comparing these profiles with the ones measured nonresonantly, we clearly recognize that each stable state on the multihysteresis corresponds to one of the excited states of the mesa, starting with the fifth excited state [see Fig. 1(f)] at low excitation power, and shifting down to the ground state at maximum power. The states are scanned back in opposite order from the ground state to the fifth excited state when the power is decreased. The spatial profile of the beam is therefore determined by the lateral confinement of the optical modes; this mechanism differs from the one based on spontaneous pattern formation due to transverse instability in a resonantly pumped planar microcavity, which was recently proposed $[41,42]$. Although the ordering in energy is respected, not all of the states appear when we increase or decrease the excitation power. On the way up, we are able to differentiate the odd from the even ( $n=2, m=2)$ four-lobe states originating from $E_{4}$; however, a single $(n=1, m=1)$ two-lobe state appears. The opposite is observed on the way down: both $(n=1, m=1)$ states are scanned (states $E_{2}$ and $\left.E_{3}\right)$ but a single ( $n=2, m=2)$ state appears. Finally, we observe that, whenever two steady states are allowed for a given excitation power, they have distinct spatial profiles: this demonstrates the spatial character of the multistability. Simply put, we can switch the transverse profile of the laser beam when transmitted through the mesa only by varying the power, while keeping the wavelength fixed.

Additional features are also present on this multistability curve. First, apart from the three upward thresholds, we observe a smoother nonlinearity on the $E_{4}$ four-lobe state at around $4 \mathrm{~mW}$, right after the first upward threshold. As opposed to the other thresholds, no bistability is observed when ramping the power up and down this nonlinearity (not shown), but we do measure a change of the spatial profile between the odd and even solution of the $(n=2, m=2)$. Second, when comparing the transverse profile of the $E_{2}$ state before the last upward threshold and after the first downward threshold, we notice a slight rotation of the symmetry axis as we approach the upward threshold. A rotation of the spatial pattern was shown to occur through coherent control by imprinting the phase of the input beam [43]. In our case, the observed phenomena originates from the cross interactions between polaritons in different modes, as explained in Sec. IV. Near the upward threshold, a state that has almost blue shifted up to the laser may induce a slight rotation of the spatial profile, hence it affects the phase relation it has with the excitation laser.

As mentioned above, the existence of the polariton spatial multistability is a consequence of cross interactions. To further confirm this point, we performed direct measurement of the polariton energy shifts along different parts of the full

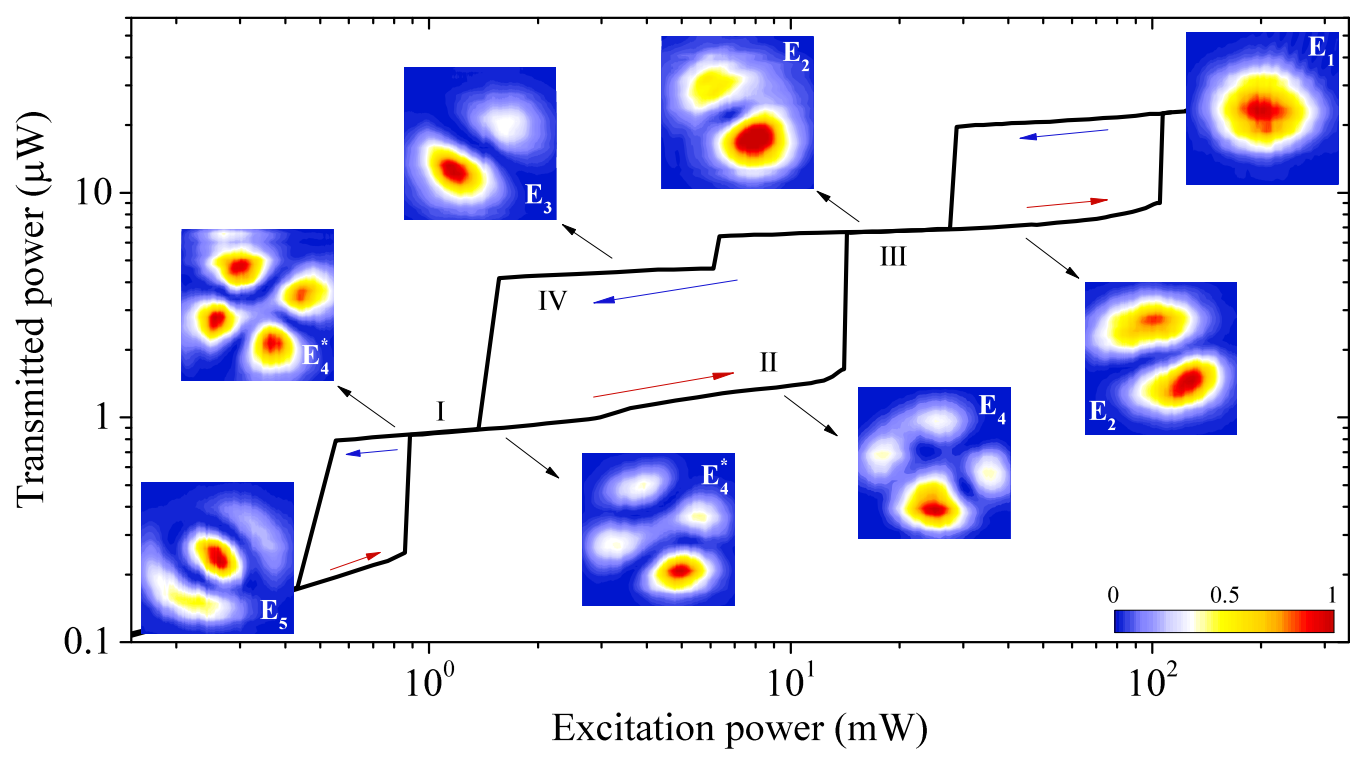

FIG. 2. Spatial multistability measured on a $9 \mu \mathrm{m}$ mesa. The black curve shows the multihysteresis cycle when we integrate spatially the transmitted signal. The red (blue) arrows indicate the path taken when increasing (decreasing) the excitation power. Each color map represents the spatial profile measured at the energy of the laser for the specific power indicated by the black arrows. Each image corresponds to a $10 \times 10 \mu \mathrm{m}$ square and the intensity is normalized from zero to one and shown in linear color scale. The roman numerals indicate regions on the curve, where the transmitted light was spectrally resolved and displayed in Fig. 3. 


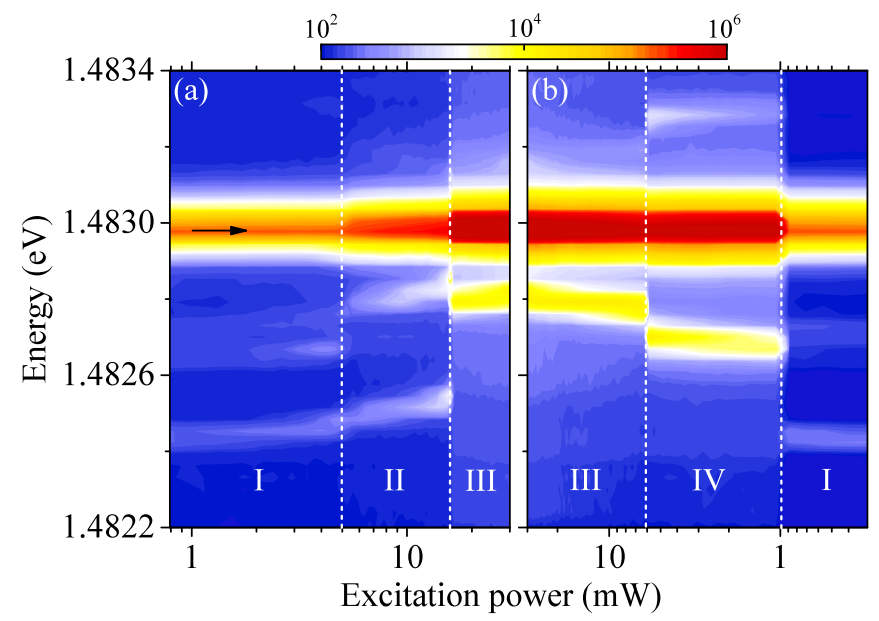

FIG. 3. PL intensity maps measured in transmission as a function of the excitation power. (a) Spectra acquired while increasing power for regions I, II, and III in Fig. 2 and (b) following regions III, IV, and back to I for decreasing power. The black arrow indicates the energy position of the excitation laser. The lower intensity lines at a lower energy correspond to states that have not reached the laser, whereas the ones at higher energy are states expelled from the resonance. The dashed white lines indicate the position of the upper (a) and lower (b) thresholds in Fig. 2.

multihysteresis cycle (see roman numerals in Fig. 2) for a given slice in the $x$ direction of the spatial profile (defined by the slit of the spectrometer). For example, Fig. 3(a) presents the spectral evolution when the excitation power is increased starting after the first threshold upward (I), passing the small nonlinearity (II), and crossing the second upward threshold to reach region (III). Figure 3(b) shows the same measurement while decreasing the excitation power, passing by region (IV) back to (I). The dominant intensity is always at the energy of the excitation laser, and the white dashed lines indicates the thresholds that are found in Fig. 2. In all these PL spectra, one clearly identifies at least one mode much weaker in intensity (two orders of magnitude) and below the laser energy; moreover, at each threshold on the multistability curve, we observe an energy jump of each of these lower energy states. If we perform a tomography of the lowest energy state on either side of the thresholds up or down (not shown), we find that its transverse profile always corresponds to that of the ground state, while the spatial profile of the transmitted laser changes according to Fig. 2.

Two mechanisms involved in the multistability are evidenced with these measurements. First, when analyzing the upward thresholds, we see that due to polariton-polariton self-interaction, the polariton state closest in energy to the laser blue shifts up to the laser energy, where a sharp increase in population occurs (as for polariton bistability). A drastic switching of the mode profile occurs at this point. Second, when this threshold occurs, all the lower energy states experience a sudden blue shift, indicating a cross interaction with the population of the state resonant with the laser. A reverse mechanism is responsible for the sudden redshifts when the excitation power is decreased and one of the state suddenly unlocks from the laser. Even though the nonlinearity measured on the multistability at $4 \mathrm{~mW}$ in Fig. 2 is weak, we clearly observe an energy jump, which confirms the measured switching of the modes. Finally, the slight variation of the threshold positions measured in PL compared to Fig. 2 is caused by the sensitivity of the experiment to intrinsic noise, especially close to the thresholds. Since we need a longer integration time to acquire the spectra compared to the spatially integrated measurements with the power meter (instantaneous value), we increase the probability of the system to undergo a noise-induced jump [32].

We are able to identify not only polariton states below the laser, but as seen between the two thresholds in Fig. 3(b), we are able to observe polariton modes above the energy of the laser. In order to follow what happens to an upper state once a lower state's spatial profile becomes dominant, we excite the sample in linear polarization and detect in cross polarization. In that situation, the overall behavior of the spatial multistability
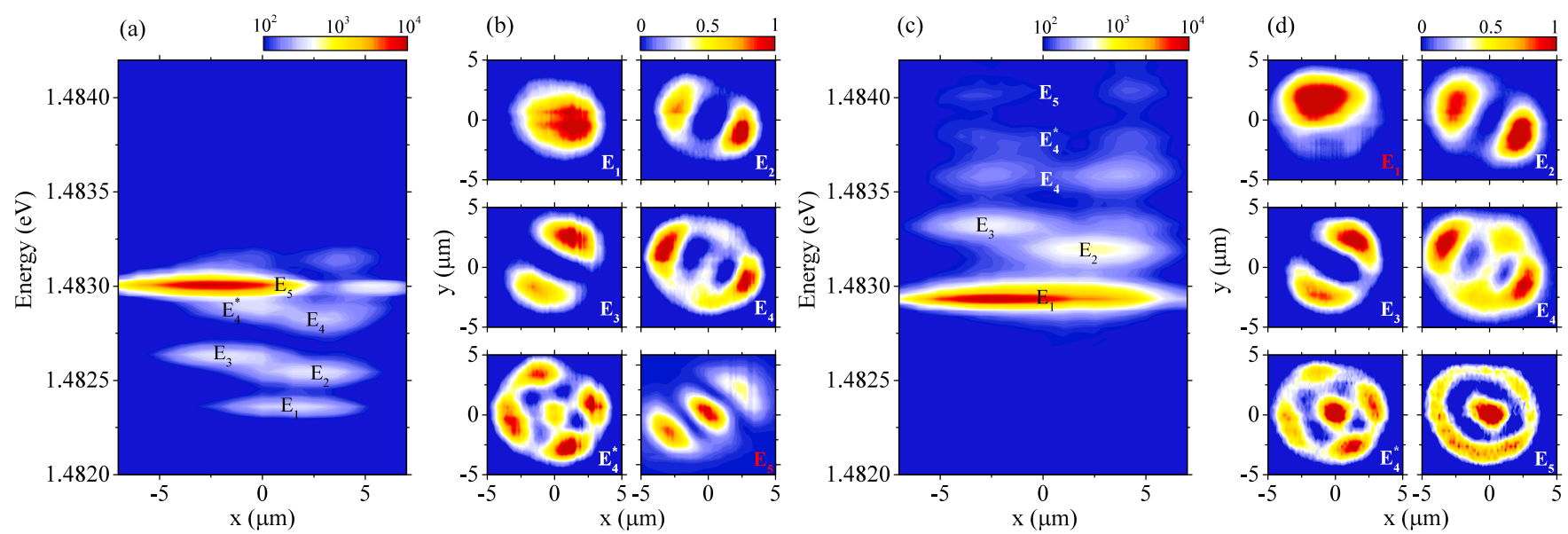

FIG. 4. (a) PL intensity as a function of position and energy for low excitation power (bottom of the multistability curve) for a given slice in the $x$ direction of the spatial profile. (b) Tomography for each state as indicated by the labels $E_{i}$ (a). (c) and (d) The same as (a) and (b) but for maximum excitation power (top of the multistability curve). For (a) and (c) the color map is in log scale, whereas for (b) and (d) the intensities are normalized and shown in linear color scale. The labels $E_{i}$ in red indicate the tomography at the energy of the laser. For each panel, the excitation laser is linearly polarized and the detection is done in cross polarization. 
is unchanged, and allows us to filter out the dominant intensity from the state at the excitation laser energy (by about four orders of magnitude) and to look for weak signals from the other states. Figures 4(a) and 4(c) present PL intensity maps for low and maximum excitation power, respectively, and for a given slice in the $x$ direction of the spatial profile. In both cases we clearly identify a series of polariton states either below the excitation laser energy (the highest intensity on the maps) for low power or above it for high excitation power. In order to confirm the nature of these states, we show the transverse spatial profiles for each of them, as indicated by the roman numerals in Figs. 4(b) and 4(d) for low and high excitation power. Notice that in both cases the energy ordering of these states is conserved independently from their relative position with respect to the excitation laser and corresponds to the energy levels measured nonresonantly as shown in Fig. 1.

With these results we can easily understand how the modes evolve during the spatial multistability. Whenever a polariton mode is blue shifted at the energy of the laser, we measure a sharp increase of the transmitted intensity accompanied by a switching of the transverse profile and correlated blue shifts for the other energy states. When a state is pulled by the laser and gets locked, it expels the previous mode above the laser energy. As explained below, the repulsive cross interactions between the polariton modes prevents them to cross each other; therefore, we observe the same ordering of the modes in energy at any excitation power.

\section{THEORETICAL MODEL}

The rich physics of microcavity polaritons builds upon exciton-exciton interactions. Numerous examples of these interactions have been studied, ranging from polariton condensate interacting with an exciton reservoir in nonresonant excitation experiments [44-49] to spinor interactions in resonant [50-55] or nonresonant excitation [56,57]. They are, however, different in nature from the spinor effects induced by the effective magnetic field caused by TE-TM splitting of the microcavity mode [58-60] or by the birefringence field induced by disorder $[61,62]$. In all these studies the interaction is essentially between two modes or components that can be either the condensate with a reservoir or interaction with opposite spins for the spinor case. Such interactions have been analyzed through the Gross-Pitaevskii formalism at the mean field level. In view of the success of these approaches to study polariton-polariton interactions, we derive a similar approach based on a set of coupled differential equations, which takes into account interactions between $N$ modes. Since each polariton mode has its own cavity detuning, it has a specific excitonic fraction (see Table I) and an energy blue shift of its own. As a consequence, a set of coupled equations in the polariton basis will not fully take into account the polariton-polariton interactions as a function of the excitation power and also the pumping efficiency (which varies according to the photonic fraction); it would need to be solved iteratively. All of this can be avoided by describing the system in the exciton-photon basis, which directly renormalizes the components of the polariton modes as a function of the excitation power.
To derive our mean field coupled equation system, we start with the usual exciton-photon Hamiltonian [63-65]:

$$
\begin{aligned}
H= & \int d \mathbf{r}\left[\hat{\psi}_{x}^{\dagger}\left(E_{x}-\frac{\hbar^{2} \nabla^{2}}{2 m_{x}}\right) \hat{\psi}_{x}+\hat{\psi}_{c}^{\dagger}\left(E_{c}-\frac{\hbar^{2} \nabla^{2}}{2 m_{c}}\right) \hat{\psi}_{c}\right. \\
& \left.+\frac{\Omega}{2}\left(\hat{\psi}_{x}^{\dagger} \hat{\psi}_{c}+\hat{\psi}_{x} \hat{\psi}_{c}^{\dagger}\right)+\frac{g_{0}}{2} \hat{\psi}_{x}^{\dagger} \hat{\psi}_{x}^{\dagger} \hat{\psi}_{x} \hat{\psi}_{x}\right],
\end{aligned}
$$

where $\hat{\psi}_{x}^{\dagger}$ and $\hat{\psi}_{c}^{\dagger}$ are exciton and cavity photon creation field operators. The Hamiltonian can be decomposed into four parts: the first two are the kinetic terms for the exciton and photon, the third is the light-matter interaction, and the last is the exciton-exciton interaction. For simplicity we do not consider the effect of quasimode coupling nor the saturation of the exciton oscillator strength [66]. We also assume circular symmetry of the system, which allows us to expand the photon operator in modes corresponding to solutions of Maxwell equations for a circular trap, namely Bessel functions of first kind and order $m: \hat{\psi}_{c}=\sum_{n, m} c_{n, m} J_{m}\left(\kappa_{n, m} r\right) e^{i m \phi} \hat{b}_{n, m}$, where $\kappa_{n, m}$ is fixed by the boundary conditions of the mesa and gives the energy of the confined photon states. The symmetry of the system implies the conservation of the angular quantum number in the light-matter coupling [35] indicating that we should describe the exciton field operator using the same expansion in Bessel functions for the center of mass part of the exciton wave function as $[35,36,64]$

$$
\hat{\psi}_{x}=\sum_{\{v, m\}} c_{\nu, m} e^{i m \phi} J_{m}\left(k_{v, m} r\right) \hat{x}_{v, m},
$$

where the $J_{m}\left(k_{v, m} r\right)$ 's are again Bessel functions of first kind and order $m, k_{v, m}$ is an exciton eigenmomentum, $\hat{x}_{v, m}$ is the annihilation operator for an exciton with angular quantum number $m$, and the sum is over sets of $\{v, m\}$ indices, which are determined by the boundary conditions. The index $v$ has a similar meaning as the radial quantum number for the photon as it sets the condition on the exciton momenta $k_{v, m}$ through the boundary condition of the system [36]. Nevertheless, the exciton motion is not affected by the mesa, and the boundary conditions are then defined by the quantization area chosen for the computation [35]. As a result, the exciton energy is barely affected and assumed to be single valued, which is consistent with the usual flat exciton dispersion measured in microcavities. Using Eq. (2) we can rewrite the light-matter Hamiltonian to properly consider the strong coupling between an exciton and a photon of the same angular quantum number. In principle, the breaking of $k$ symmetry in the mesa allows for light-matter coupling between an exciton in any state $n$ and a photon in any state $n^{\prime}$ with the same $m[35,36]$. Such a coupling would create numerous polariton modes for which a single exciton state couples with photons of various spatial profiles, resulting in an in-plane polariton distribution composed of all of them (weighted by their respective photon fraction). Since this type of coupling is not realized experimentally, we therefore use a single index $i$ to identify each allowed pair of quantum numbers $(n, m)$ to an exciton or a photon state. Furthermore, to simplify the calculation, we do not compute the full spatial evolution of the system and assume that for each mode $i$, there is a corresponding spatial profile. Finally, we can rewrite the system Hamiltonian accounting for the existence 
of multiple confined cavity modes:

$$
\begin{aligned}
H= & \sum_{i} E_{x i} \hat{x}_{i}^{\dagger} \hat{x}_{i}+\sum_{i} E_{c i} \hat{b}_{i}^{\dagger} \hat{b}_{i}+\frac{1}{2} \sum_{i} \Omega_{i}\left(\hat{x}_{i}^{\dagger} \hat{b}_{i}+\hat{x}_{i} \hat{b}_{i}^{\dagger}\right) \\
& +\frac{1}{2} \sum_{i, j, k, l} g_{i j k l} \hat{x}_{i}^{\dagger} \hat{x}_{j}^{\dagger} \hat{x}_{k} \hat{x}_{l},
\end{aligned}
$$

where $\hat{b}_{i}^{\dagger}$ is the creation operator for a cavity photon in mode $i$, $E_{c i}$ is its associated energy, and $E_{x i}$ is the energy of the exciton of state $i$. The coupling constant between the $i$ th exciton and photon modes $\Omega_{i}$ depends on the oscillator strength of the exciton as well as on the spatial overlap between the exciton and the photon. For the case of perfectly circular 9 $\mu \mathrm{m}$ mesas, the change of $\Omega_{i}$ and $E_{x i}$ between the modes is negligibly small, and therefore assumed they are constant. Following the same approach as in Ref. [65], it can be shown that only the terms conserving the number of particles in the initial and final states are important if we limit ourselves to first order perturbation theory. Therefore, the summation over four indices in Eq. (3) is replaced by a summation over two, such that $g_{i j k l} \rightarrow g_{i j}$ and $\hat{x}_{i}^{\dagger} \hat{x}_{j}^{\dagger} \hat{x}_{k} \hat{x}_{l} \rightarrow \hat{x}_{i}^{\dagger} \hat{x}_{j}^{\dagger} \hat{x}_{i} \hat{x}_{j}$. Then, using Heisenberg's equation we derive the equation of motion for each exciton and photon operator, which, following the usual mean field approximation and assuming $[67,68]$ $\left\langle\hat{x}_{i}^{\dagger} \hat{x}_{i} \hat{x}_{j}\right\rangle \cong\left\langle\hat{x}_{i}^{\dagger} \hat{x}_{i}\right\rangle\left\langle\hat{x}_{j}\right\rangle$ and $\left\langle\hat{x}_{i}^{\dagger} \hat{x}_{i}^{\dagger}\right\rangle=\left\langle\hat{x}_{j} \hat{x}_{j}\right\rangle=0 \forall(i, j)$, gives rise to a set of coupled differential equations:

$$
\begin{aligned}
& i \hbar \dot{\chi}_{i}=\left(E_{x}-i \gamma_{x}+\sum_{j} g_{i j}\left|\chi_{j}\right|^{2}\right) \chi_{i}+\frac{\Omega_{i}}{2} \phi_{i}, \\
& i \hbar \dot{\phi}_{i}=\left(E_{c i}-i \gamma_{c i}\right) \phi_{i}+\frac{\Omega_{i}}{2} \chi_{i}+f_{\text {ext }},
\end{aligned}
$$

where $\chi_{i}=\left\langle\hat{x}_{i}\right\rangle$ and $\phi_{i}=\left\langle\hat{b}_{i}\right\rangle$ are the $i$ th exciton polarization and photon mean field, respectively, and $\gamma_{x}\left(\gamma_{c i}\right)$ is their respective homogeneous linewidth. For a perfectly circular mesa, the exciton-exciton interaction terms are $g_{i j}=$ $2^{\alpha} g_{0} \int d \mathbf{r}\left|c_{i}\right|^{2}\left|c_{j}\right|^{2}\left|J_{i}(k r)\right|^{2}\left|J_{j}(k r)\right|^{2}$ and $\alpha=0$ if $i=j$ and $\alpha=1$ if $i \neq j$. Since the mesa studied is slightly elliptical, we instead consider the $g_{i j}$ as fitting parameters. Finally, the external driving field of energy $E_{l}$ which pumps each photon mode is defined as $f_{\text {ext }}=\sqrt{I_{0}} \exp \left(-i E_{l} t / \hbar\right)$. Equations (4) can be understood as a multimode generalization of the Gross-Pitaevskii equation in the exciton-photon basis $[33,69]$. These equations include the usual population exciton-exciton self-interaction terms $g_{i i}$ but also cross-interaction terms $g_{i j}$ that couple the exciton polarization of state $i$ with the population of state $j$. These terms are the ones responsible for the correlated blue shifts at each threshold as shown in Fig. 3. Note that a similar set of equations can also be obtained in the case of an exciton state coupled to a single photon mode when two pumping beams are used at different energies [18] or in the case of a polariton double well system [70], even if the cross-interaction terms were neglected in the latter case due to the specific geometry of the system.

The result of the simulation is shown in Fig. 5(a), where the sum of all photon field intensities $\left|\phi_{i}\right|^{2}$ is plotted as a function of the excitation power. For the simulation, we consider the five cavity modes associated with the polariton modes measured nonresonantly (see Table I), meaning that one of the $(n=$
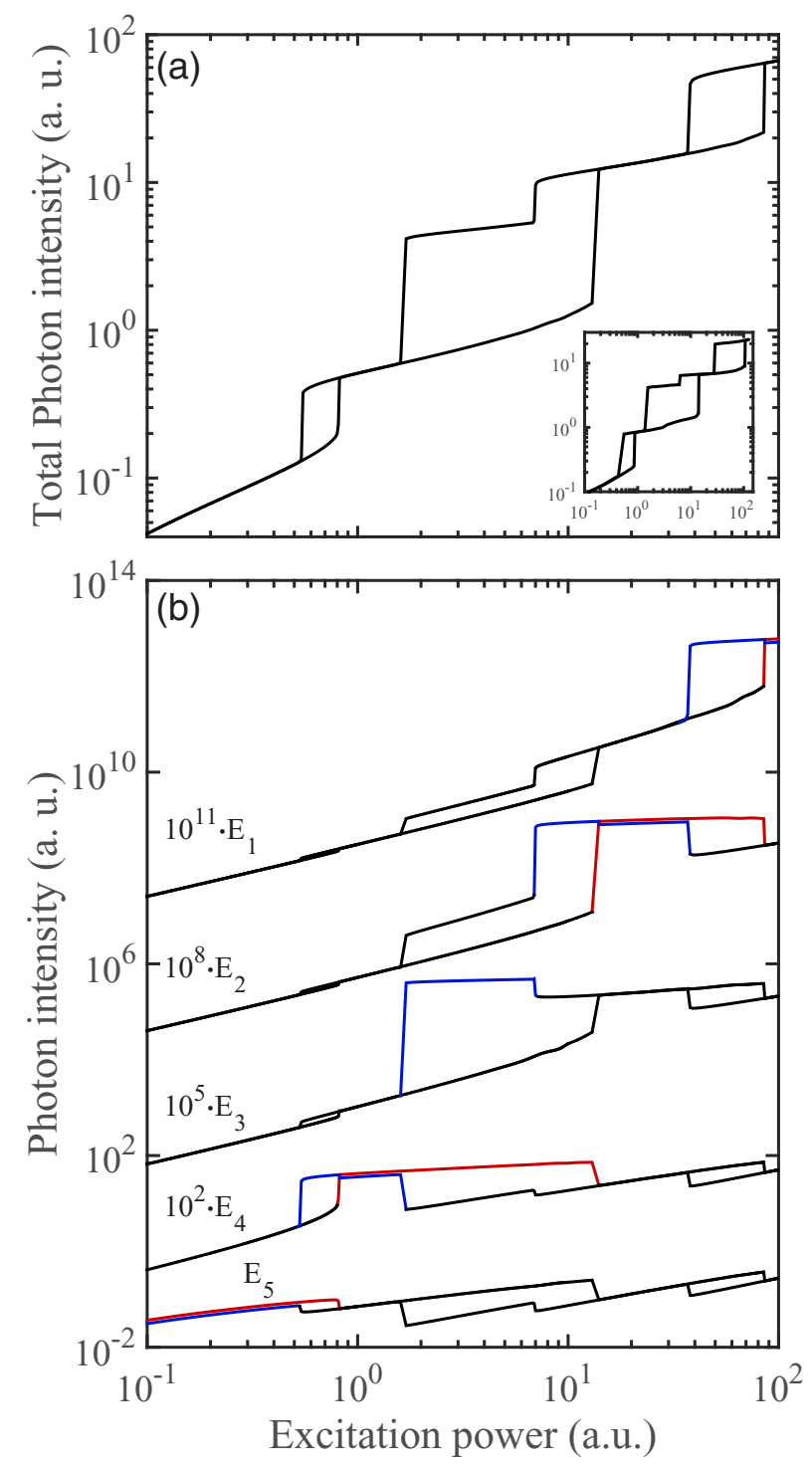

FIG. 5. (a) Simulation of the multihysteresis cycle. The simulation considers five polariton modes with the laser in resonance with the fifth one. The inset shows the experimental data as presented in Fig. 2 for comparison. (b) Calculated multihysteresis curve for each of the five cavity photon modes included in the simulation. $E_{1}$ is the cavity mode associated with the polariton ground state. Each curve has been shifted by a fixed multiplication factor given in the figure. The red (blue) parts highlighted on each curve indicates the state of highest intensity for increasing (decreasing) excitation power. Adding each red and blue fragment gives the final curve plotted in (a).

$2, m=2$ ) four-lobe states is not included. The laser energy is at the energy of fifth state $E_{5}$. We see a very nice agreement when comparing the simulation with the measured multistability in Fig. 2(a), which is reproduced in the inset of Fig. 5(a). For the same range of excitation power, the simulation shows a higher photon intensity compared to the experimental data. This is likely caused by saturation effects which are not considered in the simulation.

In order to limit the number of free parameters in the simulation, we assumed that the $g_{i i}$ 's were different from one another and that the first off-diagonal terms $g_{i j=i+1}$ 's were identical such that $g_{12}=g_{23}=g_{34}=g_{45}$ and $g_{i j}=g_{j i}$. All 
TABLE II. Values of the interaction constants $g_{i j}$ and of the homogeneous linewidths for the five cavity and exciton modes used for the simulation. The $g_{i j}$ are given in units of the scaling parameter $g_{0}=3.8 \times 10^{-4} \mathrm{meV} /$ density, where the density is fixed to one.

\begin{tabular}{llll}
\hline \hline \multicolumn{2}{c}{$\begin{array}{c}\text { Interaction constants } \\
g_{i j}\left(g_{0}\right)\end{array}$} & \multicolumn{2}{c}{$\begin{array}{c}\text { Linewidths } \\
(\mu \mathrm{eV})\end{array}$} \\
\hline$g_{11}$ & 0.355 & $\gamma_{c 1}$ & 55 \\
$g_{22}$ & 0.629 & $\gamma_{c 2}$ & 55 \\
$g_{33}$ & 0.629 & $\gamma_{c 3}$ & 32 \\
$g_{44}$ & 1.000 & $\gamma_{c 4}$ & 65 \\
$g_{55}$ & 1.000 & $\gamma_{c 5}$ & 65 \\
$g_{12}$ & 0.140 & $\gamma_{x}$ & 20 \\
$g_{13}$ & 0.100 & - & - \\
\hline \hline
\end{tabular}

other $g_{i j}$ 's are fixed to a single value because the accuracy of the simulation in that case did not allow us to justify otherwise. Finally, we allowed for a slight variation of the cavity mode linewidths to adjust the positions of the lower thresholds, as they are most sensitive to this value. The values of the parameters used for the simulation are given in Table II. The unequal number of thresholds for increasing and decreasing power can only be reproduced with $g_{12} \neq 0$. This is a clear indication that, although the polariton modes are initially independent (only a single exciton mode for a single photon mode), the polaritons in different modes interact together through the exciton-exciton interaction. The small size of the first hysteresis loop compared to the other two is the result of the small laser detuning which barely satisfies the criteria for bistability $\left(\Delta_{\text {laser }}>\sqrt{3} \gamma_{c 3}\right)$ [23].

Figure 5(b) shows the individual mode intensities as a function of the excitation power in order to understand which of the photon modes dominates in the multistability. Each curve is offset by a multiplication constant (given in the figure) to better visualize the simulation, even though it hides their relative intensities. To circumvent this visual inaccuracy, we highlight in red (blue) on each curve the state of highest intensity at each increasing (decreasing) power value. Then, adding all the red and blue fragments separately reproduces the multistability curve in Fig. 5(a). In this way we highlight that at each threshold, the dominant mode alternates, keeping an order that is given by the energy ordering at low power, which reproduces well our findings.

From this figure the influence of the cross interactions can easily be seen as, each time one of the modes becomes dominant, all the other modes jump as well. If the mode energy is below the dominant one, then its intensity increases without overcoming it. If the mode was previously locked to the laser, then its intensity drops. This explains why we measure a single transverse profile at the energy of the laser and why the other polariton modes are orders of magnitude weaker in intensity as shown in Fig. 3. By considering Eq. (4), we conclude that any abrupt jump in photon mode intensity will cause a jump in exciton population resulting in a jump in energy as observed in Fig. 3. Finally, the simulation clearly shows that both $E_{2}$ and $E_{3}$ jump at the second upper threshold and that $E_{2}$ becomes the dominant one, as observed in the experiment. In the simulation, the exciton-exciton self-interaction terms are equal $\left(g_{22}=g_{33}\right)$, which implies that the only way for the two states to have their upward threshold at the same power is through the cross interaction $g_{23}$; for this specific simulation, it is equal to $0.22 g_{22}$. Leaving $g_{23}$ to zero would create distinct thresholds for $E_{2}$ and $E_{3}$. The identical values of $g_{22}$ and $g_{33}$ are a direct consequence of the similar spatial profile of modes $E_{2}$ and $E_{3}$ since these modes originate from the same state $(n=1, m=1)$.

Since the exact conversion of the input laser power to the photon density per mode is difficult to evaluate, the interaction constants are scaled with a parameter $g_{0}$ for an arbitrary density. It is worthwhile noting that at low power, the laser is resonant with the fifth polariton mode, and thus we do not get a separate bistable behavior due to its self-interaction. Therefore, we used $g_{44}$ as the scaling parameter and simply gave to $g_{55}$ the same value. The numerical values for the $g_{i j}$ should be interpreted as effective parameters since our model does not consider saturation effects that will decrease the coupling strength $\Omega_{i}$ and alter the excitonic fractions at high density. We also neglect polariton-phonon scattering which leads to scattering between the different polariton modes and becomes efficient at high density [39]. Nonetheless, the accuracy of the simulated predictions of the model does confirm that the main processes involved in the spatial multistability are the polariton-polariton self- and cross interactions.

\section{CONCLUSIONS}

In this paper we reported on the demonstration of spatial multistability, through resonant excitation of confined polariton modes in a $9 \mu \mathrm{m}$ mesa. By exciting the sample with a laser blue detuned in energy with respect to a set of five polariton modes, we measured a series of thresholds of the transmitted intensity when we increased or decreased the excitation power, resulting in polariton multistability. Furthermore, each stable position along the multistability curve corresponds to a specific transverse spatial profile of the transmitted laser beam, which arises from the profile of the corresponding confined polariton mode. This behavior has an important implication: the transverse spatial profile of the transmitted laser beam can simply be varied by changing its input power.

In general, the polariton bistability is driven by the polariton-polariton self-interactions mediated by the exciton; in these measurements, however, we have shown that cross interactions occur between the polariton modes and trigger the observed spatial multistability. We confirmed this mechanism by tracking the spectral shift of the polariton modes below and above the excitation laser energy and by observing that the energy jumps of the modes is concomitant with the intensity jumps of the transmitted laser beam at each threshold.

Finally, all the main features of the observed multistability have been successfully simulated using a multimode version of the Gross-Pitaevskii equations in the exciton-photon basis. The equations contained the nonlinear effects of cross interactions among different modes in order to explain the energy blue shifts, the asymmetry in the upward and downward thresholds, and the transmission of a laser beam, the profile of which switched between the polariton mode profiles. This formalism could be applied, in principle, to the study of multimode polariton-polariton interactions in a variety of confined exciton-polariton systems. 


\section{ACKNOWLEDGMENTS}

The present work is supported by the Swiss National Science Foundation under project No. 135003 and the European Research Council under project Polaritonics contract No. 291120. The polariton network is also acknowledged.
[1] H. M. Gibbs, Optical Bistability: Controlling Light with Light (Academic, London, 1985).

[2] S. Cecchi, G. Giusfredi, E. Petriella, and P. Salieri, Phys. Rev. Lett. 49, 1928 (1982).

[3] J. Nalik, W. Lange, and F. Mitschke, Appl. Phys. B 49, 191 (1989).

[4] A. Joshi and M. Xiao, Phys. Rev. Lett. 91, 143904 (2003).

[5] J. Sheng, U. Khadka, and M. Xiao, Phys. Rev. Lett. 109, 223906 (2012).

[6] C. Tamm and C. O. Weiss, J. Opt. Soc. Am. B 7, 1034 (1990).

[7] D. Wilkowski, D. Hennequin, D. Dangoisse, and P. Glorieux, Chaos Solitons Fractals 4, 1683 (1994).

[8] A. B. Coates, C. O. Weiss, C. Green, E. J. D'Angelo, J. R. Tredicce, M. Brambilla, M. Cattaneo, L. A. Lugiato, R. Pirovano, F. Prati et al., Phys. Rev. A 49, 1452 (1994).

[9] M. Brambilla, L. A. Lugiato, V. Penna, F. Prati, C. Tamm, and C. O. Weiss, Phys. Rev. A 43, 5114 (1991).

[10] M. Travagnin, M. Pinna, F. Prati, and L. A. Lugiato, Opt. Commun. 108, 377 (1994).

[11] O. El Daif, A. Baas, T. Guillet, J.-P. Brantut, R. I. Kaitouni, J. L. Staehli, F. Morier-Genoud, and B. Deveaud, Appl. Phys. Lett. 88, 061105 (2006).

[12] D. Bajoni, P. Senellart, E. Wertz, I. Sagnes, A. Miard, A. Lemaître, and J. Bloch, Phys. Rev. Lett. 100, 047401 (2008).

[13] K. Winkler, C. Schneider, J. Fischer, A. Rahimi-Iman, M. Amthor, A. Forchel, S. Reitzenstein, S. Höfling, and M. Kamp, Appl. Phys. Lett. 102, 041101 (2013).

[14] K. Winkler, J. Fischer, A. Schade, M. Amthor, R. Dall, J. Geßler, M. Emmerling, E. A. Ostrovskaya, M. Kamp, C. Schneider et al., New J. Phys. 17, 023001 (2015).

[15] J. M. Zajac, W. Langbein, M. Hugues, and M. Hopkinson, Phys. Rev. B 85, 165309 (2012).

[16] N. A. Gippius, I. A. Shelykh, D. D. Solnyshkov, S. S. Gavrilov, Y. G. Rubo, A. V. Kavokin, S. G. Tikhodeev, and G. Malpuech, Phys. Rev. Lett. 98, 236401 (2007).

[17] S. S. Gavrilov, N. A. Gippius, S. G. Tikhodeev, and V. D. Kulakovskii, J. Exp. Theor. Phys. 110, 825 (2010).

[18] E. Cancellieri, F. M. Marchetti, M. H. Szymanska, and C. Tejedor, Phys. Rev. B 83, 214507 (2011).

[19] D. V. Vishnevsky, D. D. Solnyshkov, N. A. Gippius, and G. Malpuech, Phys. Rev. B 85, 155328 (2012).

[20] O. Bozat, I. G. Savenko, and I. A. Shelykh, Phys. Rev. B 86, 035413 (2012).

[21] O. Kyriienko, E. A. Ostrovskaya, O. A. Egorov, I. A. Shelykh, and T. C. H. Liew, Phys. Rev. B 90, 125407 (2014).

[22] G. Slavcheva, A. V. Gorbach, A. Pimenov, A. G. Vladimirov, and D. V. Skryabin, Opt. Lett. 40, 1787 (2015).

[23] A. Baas, J. P. Karr, H. Eleuch, and E. Giacobino, Phys. Rev. A 69, 023809 (2004).

[24] T. K. Paraïso, M. Wouters, Y. Léger, F. Morier-Genoud, and B. Deveaud-Plédran, Nat. Mater. 9, 655 (2010).
[25] C. Adrados, A. Amo, T. C. H. Liew, R. Hivet, R. Houdré, E. Giacobino, A. V. Kavokin, and A. Bramati, Phys. Rev. Lett. 105, 216403 (2010).

[26] M. De Giorgi, D. Ballarini, E. Cancellieri, F. M. Marchetti, M. H Szymanska, C. Tejedor, R. Cingolani, E. Giacobino, A. Bramati, G. Gigli et al., Phys. Rev. Lett. 109, 266407 (2012).

[27] R. Cerna, Y. Léger, T. K. Paraïso, M. Wouters, F. MorierGenoud, M. T. Portella-Oberli, and B. Deveaud, Nat. Commun. 4, 2008 (2013).

[28] S. S. Gavrilov, A. V. Sekretenko, N. A. Gippius, C. Schneider, S. Höfling, M. Kamp, A. Forchel, and V. D. Kulakovskii, Phys. Rev. B 87, 201303 (2013).

[29] T. Boulier, M. Bamba, A. Amo, C. Adrados, A. Lemaitre, E. Galopin, I. Sagnes, J. Bloch, C. Ciuti, E. Giacobino et al., Nat. Commun. 5, 3260 (2014).

[30] H. Abbaspour, S. Trebaol, F. Morier-Genoud, M. T. PortellaOberli, and B. Deveaud, Phys. Rev. Lett. 113, 057401 (2014).

[31] H. Abbaspour, S. Trebaol, F. Morier-Genoud, M. T. Portella-Oberli, and B. Deveaud, Phys. Rev. B 91, 155307 (2015).

[32] H. Abbaspour, G. Sallen, S. Trebaol, F. Morier-Genoud, M. T. Portella-Oberli, and B. Deveaud, Phys. Rev. B 92, 165303 (2015).

[33] M. Wouters, T. K. Paraïso, Y. Léger, R. Cerna, F. MorierGenoud, M. T. Portella-Oberli, and B. Deveaud-Plédran, Phys. Rev. B 87, 045303 (2013).

[34] T. A. Fisher, A. M. Afshar, M. S. Skolnick, D. M. Whittaker, and J. S. Roberts, Phys. Rev. B 53, R10469 (1996).

[35] P. Lugan, D. Sarchi, and V. Savona, Phys. Status Solidi C 3, 2428 (2006).

[36] G. Panzarini and L. C. Andreani, Phys. Rev. B 60, 16799 (1999).

[37] R. I. Kaitouni, O. El Daif, A. Baas, M. Richard, T. Paraiso, P. Lugan, T. Guillet, F. Morier-Genoud, J. Ganière, J. Staehli et al., Phys. Rev. B 74, 155311 (2006).

[38] G. Nardin, Y. Léger, B. Pietka, F. Morier-Genoud, and B. Deveaud-Plédran, Phys. Rev. B 82, 045304 (2010).

[39] G. Grosso, S. Trebaol, M. Wouters, F. Morier-Genoud, M. T. Portella-Oberli, and B. Deveaud, Phys. Rev. B 90, 045307 (2014).

[40] T. Paraïso, D. Sarchi, G. Nardin, R. Cerna, Y. Leger, B. Pietka, M. Richard, O. El Daï, F. Morier-Genoud, V. Savona et al., Phys. Rev. B 79, 045319 (2009).

[41] M. H. Luk, Y. C. Tse, N. H. Kwong, P. T. Leung, P. Lewandowski, R. Binder, and S. Schumacher, Phys. Rev. B 87, 205307 (2013).

[42] O. A. Egorov, A. Werner, T. C. H. Liew, E. A. Ostrovskaya, and F. Lederer, Phys. Rev. B 89, 235302 (2014).

[43] R. Cerna, D. Sarchi, T. K. Paraïso, G. Nardin, Y. Léger, M. Richard, B. Pietka, O. El Daif, F. Morier-Genoud, V. Savona et al., Phys. Rev. B 80, 121309 (2009). 
[44] E. Wertz, L. Ferrier, D. D. Solnyshkov, R. Johne, D. Sanvitto, A. Lemaître, I. Sagnes, R. Grousson, A. V. Kavokin, P. Senellart et al., Nat. Phys. 6, 860 (2010).

[45] L. Ferrier, E. Wertz, R. Johne, D. D. Solnyshkov, P. Senellart, I. Sagnes, A. Lemaître, G. Malpuech, and J. Bloch, Phys. Rev. Lett. 106, 126401 (2011).

[46] G. Tosti, G. Christmann, N. G. Berloff, P. Tsotsis, T. Gao, Z. Hatzopoulos, P. G. Savvidis, and J. J. Baumberg, Nat. Phys. 8, 190 (2012).

[47] P. Cristofolini, A. Dreismann, G. Christmann, G. Franchetti, N. G. Berloff, P. Tsotsis, Z. Hatzopoulos, P. G. Savvidis, and J. J. Baumberg, Phys. Rev. Lett. 110, 186403 (2013).

[48] C. Sturm, D. Tanese, H. S. Nguyen, H. Flayac, E. Galopin, A. Lemaître, I. Sagnes, D. Solnyshkov, A. Amo, G. Malpuech et al., Nat. Commun. 5, 3278 (2014).

[49] A. Askitopoulos, T. C. H. Liew, H. Ohadi, Z. Hatzopoulos, P. G. Savvidis, and P. G. Lagoudakis, Phys. Rev. B 92, 035305 (2015).

[50] T. C. H. Liew, A. V. Kavokin, and I. A. Shelykh, Phys. Rev. Lett. 101, 016402 (2008).

[51] M. Vladimirova, S. Cronenberger, D. Scalbert, K. V. Kavokin, A. Miard, A. Lemaître, J. Bloch, D. Solnyshkov, G. Malpuech, and A. V. Kavokin, Phys. Rev. B 82, 075301 (2010).

[52] A. Amo, T. C. H. Liew, C. Adrados, R. Houdré, E. Giacobino, A. V. Kavokin, and A. Bramati, Nat. Photon. 4, 361 (2010).

[53] R. Hivet, H. Flayac, D. D. Solnyshkov, D. Tanese, T. Boulier, D. Andreoli, E. Giacobino, J. Bloch, A. Bramati, G. Malpuech et al., Nat. Phys. 8, 724 (2012).

[54] N. Takemura, S. Trebaol, M. Wouters, and B. Deveaud, Nat. Phys. 10, 500 (2014).

[55] N. Takemura, S. Trebaol, M. Wouters, M. T. Portella-Oberli, and B. Deveaud, Phys. Rev. B 90, 195307 (2014).

[56] F. Manni, K. G. Lagoudakis, T. C. H. Liew, R. André, V. Savona, and B. Deveaud, Nat. Commun. 3, 1309 (2012).
[57] H. Ohadi, A. Dreismann, Y. G. Rubo, F. Pinsker, Y. del ValleInclan Redondo, S. I. Tsintzos, Z. Hatzopoulos, P. G. Savvidis, and J. J. Baumberg, Phys. Rev. X 5, 031002 (2015).

[58] I. Shelykh, G. Malpuech, K. V. Kavokin, A. V. Kavokin, and P. Bigenwald, Phys. Rev. B 70, 115301 (2004).

[59] A. Kavokin, G. Malpuech, and M. Glazov, Phys. Rev. Lett. 95, 136601 (2005).

[60] C. Leyder, M. Romanelli, J. P. Karr, E. Giacobino, T. C. H. Liew, M. M. Glazov, A. V. Kavokin, G. Malpuech, and A. Bramati, Nat. Phys. 3, 628 (2007).

[61] K. G. Lagoudakis, T. Ostatnický, A. V. Kavokin, Y. G. Rubo, R. André, and B. Deveaud-Plédran, Science 326, 974 (2009).

[62] F. Manni, Y. Léger, Y. G. Rubo, R. André, and B. Deveaud, Nat. Commun. 4, 2590 (2013).

[63] G. Rochat, C. Ciuti, V. Savona, C. Piermarocchi, A. Quattropani, and P. Schwendimann, Phys. Rev. B 61, 13856 (2000).

[64] A. Verger, C. Ciuti, and I. Carusotto, Phys. Rev. B 73, 193306 (2006).

[65] N. Takemura, S. Trebaol, M. D. Anderson, V. Kohnle, Y. Leger, D. Y. Oberli, M. T. Portella-Oberli, and B. Deveaud, Phys. Rev. B 92, 125415 (2015).

[66] C. Ciuti, P. Schwendimann, B. Deveaud, and A. Quattropani, Phys. Rev. B 62, R4825 (2000).

[67] S. Mukamel, Principles of Nonlinear Optical Spectroscopy (Oxford University Press, New York, 1995).

[68] N. Takemura, M. D. Anderson, S. Trebaol, S. Biswas, D. Y. Oberli, M. T. Portella-Oberli, and B. Deveaud, Phys. Rev. B 92, 235305 (2015).

[69] We do not include the spin in these equations as it is done in Ref. [33]. Although straightforward to include, this would double the number of interaction constants. We would also need to double the number of photon modes as the breaking of circular symmetry in real mesas lead to linearly polarized cavity modes.

[70] D. Sarchi, I. Carusotto, M. Wouters, and V. Savona, Phys. Rev. B 77, 125324 (2008). 